\section{Moving the genetics of inflammatory bowel diseases from bench to bedside: first steps towards personalised medicine}

\author{
Stephan Brand
}

In the past decade, we have witnessed enormous progress in the understanding of the genetics of inflammatory bowel diseases (IBD), resulting in the discovery and confirmation of 163 IBD susceptibility regions by the end of $2012^{1-3}$ which is the highest number of susceptibility genes discovered for any complex disease so far. However, clinically highly relevant gene associations are still very limited, thereby also limiting the use of genetic information in the current treatment of IBD patients. The European IBDchip project ${ }^{4}$ represents a large detailed genotypephenotype analysis of IBD patients and starts to fill the gap between IBD genetics and clinically relevant information.

This European multicentre study analysed more than 1500 patients with Crohn's disease (CD) for CD susceptibility variants, identified in the meta-analysis of genome-wide association studies (GWAS) by Barrett et al, ${ }^{5}$ regarding predictors for $\mathrm{CD}$ outcomes such as disease localisation and disease behaviour. ${ }^{4}$ The main result of this study was the identification of NOD2 as the most important genetic predictor for ileal disease, ileal stenoses, fistula and CD-related surgery. This confirms previous reports showing particularly strong associations with ileal involvement, ileal stenosis and need for surgery in patients homozygous for the p.Leu1007fsX1008 NOD2 variant. $^{6}{ }^{7}$ An inadequate immune response to bacterial antigens in patients with mutated NOD2 gene resulting in chronic ileal inflammation seems to be the starting point for a sequel of events leading to ileal stenoses and thereby increasing the risk for fistula formation, ${ }^{8}$ finally requiring surgery. ${ }^{6}$

Janus kinase 2 (JAK2) was the other gene significantly associated with ileocolonic disease involvement and stenosing disease behaviour, although the disease associations with JAK2 were weaker than that with NOD2. ${ }^{4}$ JAK2 is a key component of the

Correspondence to Prof, Dr Stephan Brand, Department of Medicine II, University Hospital MunichGrosshadern, Marchioninistr. 15, Munich D-81377, Germany; stephan.brand@med.uni-muenchen.de signal transduction pathway of several cytokines including interleukin (IL)-12 and IL-23 which play a key role in the pathogenesis of CD. ${ }^{9}$ Interestingly, tofacitinib (CP-690 550), a selective oral inhibitor of the JAK family of kinases, particularly of JAK1 and JAK3, may at high doses also inhibit JAK2 and is very effective in the treatment of ulcerative colitis (UC). ${ }^{10}$

The results of the European IBDchip study ${ }^{4}$ suggest that the natural course of CD may be changed by immunosuppressive therapy which supports an early use of immunosuppressive therapy. While the use of immunosuppressives such as azathioprine within 3 years of CD diagnosis led to a strong decrease of intestinal stenoses and need for CD-related surgery, a similar decrease could not been shown for antitumor necrosis factor (TNF) agents. This is most likely related to the very low number of patients receiving anti-TNF agents within their first 3 years of $\mathrm{CD}$ diagnosis, representing only $3.3 \%$ of the patients of this study cohort. This may be related to one of the inclusion criterions, which required a long follow-up period of at least 10 years, suggesting that for many of the study patients, anti-TNF agents were not available during the time of their early disease. Based on the high efficacy of anti-TNF agents, which have been shown to be even more efficacious than azathioprine in the SONIC trial, ${ }^{11}$ a very similar effect may be hypothesised for anti-TNF antibodies.

The publication of the European IBDchip project coincides with the release of the data of the largest IBD meta-analysis of GWAS ever performed consisting of a combined total of more than 75000 IBD patients and controls. ${ }^{3}$ This GWAS meta-analysis identified 71 novel IBD susceptibility regions, thereby increasing the number of IBD susceptibility loci to $\mathrm{n}=163$. Interestingly, 110 out of these 163 IBD genes are susceptibility genes for CD and UC which is an unexpected high genetic overlap for these distinct phenotypes of IBD. However, out of all 163 IBD susceptibility genes identified, only two genes (NOD2 and IL23R) have an OR $>1.5$ for IBD susceptibility compared with healthy controls, while for many IBD genes the $\mathrm{OR}<1.2$, limiting their use in clinical practice. Whereas NOD2 represents the key $\mathrm{CD}$ risk gene involved in innate immunity, $I L 23 R$ is a key gene in adaptive immunity (figure 1). NOD2, the first identified CD risk gene, is still the strongest $\mathrm{CD}$ susceptibility gene. The clinical importance of NOD2 is nicely illustrated by a case report in which we recently described a family with all four family members being affected by $\mathrm{CD}$; all family members were carriers of NOD2 mutations. ${ }^{12}$

How may IBD genetics guide the way we will practice medicine in IBD patients in the near future? Prediction of the disease phenotype and prediction of the response to medical therapy are the obvious two main areas, in which IBD genetics may change clinical practice (figure 1). First, IBD genetics may predict the disease phenotype such as disease localisation, disease behaviour and the likelihood for extraintestinal manifestations (figure 1). The potential for such predictions has been shown in the study by Cleynen et al. ${ }^{4}$ Compared with NOD2 wild type carriers, $C D$ patients with NOD2 mutations had a threefold higher risk for a complicated CD clinical course and were almost twice as likely to undergo bowel resection. ${ }^{4}$ This risk is further exacerbated in patients homozygous for the p.Leu1007fsX1008 NOD2 variant. ${ }^{4}{ }^{6}$ However, it has to be noted that in a recent meta-analysis the predictive power of a single NOD2 variant was low, while the presence of two NOD2 mutations had $98 \%$ specificity for complicated $\mathrm{CD}^{13}$ which may support more 'aggressive' therapeutic strategies in this particular patient subgroup. Certain gene variants may also help to predict the likelihood for developing extraintestinal manifestations (figure 1). For example, carriers of the rs2476601 PTPN22 variant had an almost twofold increased risk for extraintestinal manifestations. ${ }^{4}$

The second important area of using IBD genetics in clinical practice will be IBD therapy (figure 1). In particular, the response to therapy and the risk for side effects are major areas of interest. It is likely that in addition to known IBD susceptibility genes, other genes will influence the IBD phenotype and have an impact on pharmacogenomics such as shown for the gene encoding thiopurine $\mathrm{S}$-methyltransferase as a major modulator of the metabolism of thiopurines. In contrast, predictors for the response to anti-TNF therapy are currently more limited. In a pilot study, we demonstrated certain IL23R gene variants as modulators of the response to anti-TNF therapy 


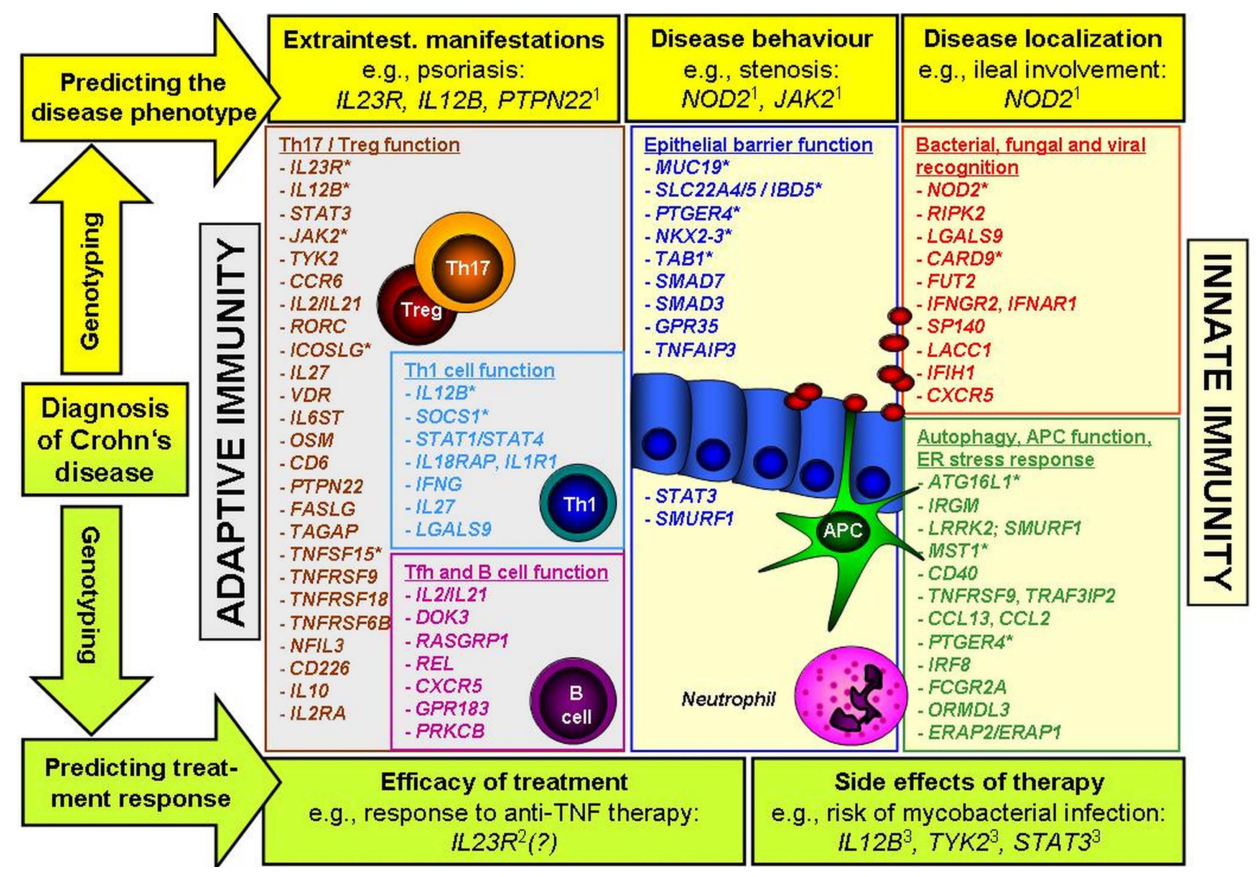

Figure 1 The main susceptibility genes for Crohn's disease (CD) and their role in predicting the disease phenotype and the treatment response. Currently, the detailed functional mechanisms by which risk variants modulate CD susceptibility are unknown for most of the genes shown in this figure. Therefore, the grouping is largely based on biological properties described for these genes so far. Genes may have activating or inhibiting effects on the cell types under which they are listed. Genes with a presumably more important role in innate immunity are shown in light yellow boxes, while genes with a presumably more important role in adaptive immunity are shown in grey boxes. *Genes marked with an asterisk are major CD risk variants which explain more than $1 \%$ of the total variance explained by all loci for CD based on the study by Jostins et al. ${ }^{3}$ (1) These associations with the CD phenotype were shown in the study by Cleynen et al. ${ }^{4}$ (2) We demonstrated IL23R variants as predictors of the response to anti-TNF therapy in ulcerative colitis; however, this needs confirmation in $\mathrm{CD}^{8}{ }^{8}(3)$ The associations with susceptibility to mycobacterial infection are highlighted in the study by Jostins et al. ${ }^{3} \mathrm{APC}$, antigen presenting cell; ER, endoplasmic reticulum; NKT, natural killer cells; Th, T helper cell; Tfh, follicular helper T cell; Treg, regulatory T cell.

in UC patients. ${ }^{14} I L 23 R$ is also a risk gene for ankylosing spondylitis which is more common in IBD patients than in unaffected controls. Anti-TNF agents are approved for IBD and ankylosing spondylitis, thereby illustrating an example where genotyping may help to identify extraintestinal manifestations and guide the selection of therapy.

However, IBD genetics is only one tool to predict the disease behaviour and disease course. To refine the prognostic power of prediction models, other biomarkers such as serological and microbiological markers, additional risk factors (eg, smoking, young age at disease onset) and the type of medical therapy have to be included in future risk models. The study of Cleynen et $a l^{4}$ shows also the current limits of IBD genetics and phenotype prediction. With the exception of NOD2, prediction models based on CD susceptibility variants other than NOD2, had limited predictive ability for specific disease phenotypes. ${ }^{4}$ For example, in logistic regression models, the area under the receiver operating characteristic curve was $61 \%$ for penetrating CD behaviour and $72 \%$ for complicating disease behaviour, ${ }^{4}$ limiting the use of these prediction models in clinical practice. Although the study by Cleynen et $a l^{5}$ was based on the results of a GWAS meta-analysis, the study itself did not employ GWAS technology thereby many clinically useful predictors may not have been detected in this study. ${ }^{4}$

In summary, the European IBDchip study demonstrates the potential for predicting CD phenotypes. ${ }^{4}$ The strongest genetic predictor for a complicated disease course and the need for surgery is the NOD2 variant, ${ }^{4}$ although only in the case of two mutated NOD2 alleles there is sufficient specificity for phenotype predictions. ${ }^{13}$ The greatest risk for developing ileal stenosis requiring surgery has been shown for CD patients homozygous for the p.Leu1007fsX1008 NOD2 variant $^{6} 7$ which would be a candidate patient group for early immunosuppressive therapy which may change the natural course of $\mathrm{CD}$, resulting in fewer surgical interventions. ${ }^{4}$ The overall OR for complicated disease behaviour was 4.87 for CD patients homozygous for the p.Leu1007fsX1008 NOD2 mutation. ${ }^{4}$ However, in Europe, this subgroup represents only less than $3 \%$ of all CD patients which, together with the limited prognostic ability of current phenotype prediction models and the low OR of most IBD risk genes regarding disease susceptibility limits the use of IBD genotyping in current clinical practice. Moreover, the results of the study by Cleynen et al have to be replicated in an independent cohort. However, even replicated gene markers with highly significant ORs may be insufficient classifiers for a complex disease such as $\mathrm{CD}$, given the strong contribution of non-genetic environmental factors such as microbiota, viruses, hygiene (particularly during early childhood), smoking, diet, appendectomy and the use of medical therapy such as antibiotics and non-steroidal anti-inflammatory agents. ${ }^{15}$ In addition, even the large number of identified IBD susceptibility risk genes explains only a minority of the variance in disease risk, suggesting that in addition to environmental factors rarer genetic variants, which are not captured by GWAS, are substantial contributors to the IBD pathogenesis. Considering the major ongoing efforts of the International Inflammatory Bowel Disease Genetics Consortium and the implementation of GWAS, next-generation sequencing and fine-mapping studies, it is likely that in the 
foreseeable future a panel of genes and other biomarkers including profiling of microbiota and clinical disease characteristics such as smoking status will allow a more detailed prediction of the disease course and the efficacy and risks of medical therapy, thereby providing a personalised therapeutic approach for IBD patients (figure 1).

Funding SB was supported by grants from the Deutsche Forschungsgemeinschaft (DFG) (BR 1912/6-1) and the Else Kröner-Fresenius-Stiftung (Else Kröner-Exzellenzstipendium 2010_EKES.32)

Competing interests None.

Provenance and peer review Commissioned; externally peer reviewed.

To cite Brand S. Gut 2013;62:1531-1533.

Published Online First 30 January 2013

\section{CLinked}

http://dx.doi.org/10.1136/gutjnl-2011-300777

Gut 2013;62:1531-1533.

doi:10.1136/gutjnl-2012-304151

\section{REFERENCES}

1 Franke A, McGovern DP, Barrett JC, et al. Genome-wide meta-analysis increases to 71 the number of confirmed Crohn's disease susceptibility loci. Nat Genet 2010;42:1118-25.

2 Anderson CA, Boucher G, Lees CW, et al. Meta-analysis identifies 29 additional ulcerative colitis risk loci, increasing the number of confirmed associations to 47. Nat Genet 2011;43:246-52.

3 Jostins L, Ripke S, Weersma RK, et al. Host-microbe interactions have shaped the genetic architecture of inflammatory bowel disease. Nature 2012;491: 119-24.

4 Cleynen I, Gonzalez JR, Figueroa C, et al. Genetic factors conferring an increased susceptibility to develop Crohn's disease also influence disease phenotype: results from the IBDchip European Project. Gut 2013;62:1556-65.

5 Barrett JC, Hansoul S, Nicolae DL, et al. Genome-wide association defines more than 30 distinct susceptibility loci for Crohn's disease. Nat Genet 2008;40:955-62.

6 Seiderer J, Brand S, Herrmann KA, et al. Predictive value of the CARD15 variant 1007fs for the diagnosis of intestinal stenoses and the need for surgery in Crohn's disease in clinical practice: results of a prospective study. Inflamm Bowel Dis 2006;12:1114-21.

7 Seiderer J, Schnitzler F, Brand S, et al. Homozygosity for the CARD15 frameshift mutation 1007fs is predictive of early onset of Crohn's disease with ileal stenosis, entero-enteral fistulas, and frequent need for surgical intervention with high risk of re-stenosis. Scand I Gastroenterol 2006;41:1421-32.

8 Jürgens $\mathrm{M}$, Brand S, Laubender RP, et al. The presence of fistulas and NOD2 homozygosity strongly predict intestinal stenosis in Crohn's disease independent of the IL23R genotype. J Gastroenterol 2010;45:721-31.

9 Brand S. Crohn's disease: Th1, Th17 or both? The change of a paradigm: new immunological and genetic insights implicate Th17 cells in the pathogenesis of Crohn's disease. Gut 2009;58:1152-67.

10 Sandborn WJ, Ghosh S, Panes J, et al. Tofacitinib, an oral Janus kinase inhibitor, in active ulcerative colitis. N Engl J Med 2012;367:616-24.

11 Colombel JF, Sandborn WJ, Reinisch W, et al. Infliximab, azathioprine, or combination therapy for Crohn's disease. $N$ Engl J Med 2010;362:1383-95.

12 Schnitzler F, Seiderer J, Stallhofer J, et al. Dominant disease-causing effect of NOD2 mutations in a family with all family members affected by Crohn's disease. Inflamm Bowel Dis 2012;18:395-6.

13 Adler J, Rangwalla SC, Dwamena BA, et al. The prognostic power of the NOD2 genotype for complicated Crohn's disease: a meta-analysis. Am J Gastroenterol 2011;106:699-712.

14 Jürgens M, Laubender RP, Hartl F, et al. Disease activity, ANCA, and IL23R genotype status determine early response to infliximab in patients with ulcerative colitis. Am J Gastroenterol 2010;105:1811-19.

15 Kaser A, Zeissig S, Blumberg RS. Genes and environment: how will our concepts on the pathophysiology of IBD develop in the future? Dig Dis 2010;28:395-405. 


\title{
GUT Moving the genetics of inflammatory bowel diseases from bench to bedside: first steps towards personalised medicine
}

Stephan Brand

Gut 2013 62: 1531-1533 originally published online January 30, 2013 doi: 10.1136/gutjnl-2012-304151

Updated information and services can be found at:

http://gut.bmj.com/content/62/11/1531

\begin{abstract}
These include:
References This article cites 15 articles, 2 of which you can access for free at: http://gut.bmj.com/content/62/11/1531\#BIBL

Email alerting

Receive free email alerts when new articles cite this article. Sign up in the service box at the top right corner of the online article.
\end{abstract}

\section{Notes}

To request permissions go to:

http://group.bmj.com/group/rights-licensing/permissions

To order reprints go to:

http://journals.bmj.com/cgi/reprintform

To subscribe to BMJ go to:

http://group.bmj.com/subscribe/ 